\title{
In die Zange genommen
}

Im Zusammenhang mit den grundlegenden gesellschaftskritischen Bewegungen, die sich um 1968 in Europa etablieren, setzt in Westdeutschland auch die Kritik am modernen Wohnungsbau des Wiederaufbaus ein. Um diese Kritik besser zu verstehen lohnt es sich, parallel die Diskussion um den Altbaubestand der Gründerzeit zu beleuchten. Die These, die ich hier diskutieren möchte, lautet: Die Wiederentdeckung des Altbaubestandes und die Kritik am modernen Wohnungs- und Siedlungsbau sind zwei Seiten derselben Medaille. Diese These beruht wesentlich auf der Überzeugung, dass beide Bewegungen, sowohl die Abwertung des Altbaubestandes und die Begeisterung für den modernen Siedlungsbau als auch die folgende Umkehrung, aus dem zeitgenössischen gesellschaftlichen, wirtschaftlichen und politischen Kontext heraus verstanden werden müssen. Ich unterstelle der Kritik am Siedlungsbau der Moderne in diesem Beitrag, dass sie genau dies weder 1968 noch in der Zeit zwischen 1968 und heute getan hat. Meiner Ansicht nach gibt die Kritik am Siedlungsbau der Moderne primär die Stimme der akademischen Mittelschicht wieder, die sie in die Presse und in den Architektur- und Planungsdiskurs eingebracht hat und diesen bis heute dominiert.

\section{Siedlungsbau im Kontext der Nachkriegszeit}

Das Wohnen in der Stadt war schon vor den Kriegszerstörungen denkbar schlecht, weil das explosionsartige Stadtwachstum der Industrialisierung nicht bewältigt worden war. Die Kriegszerstörungen verschärften also einen eigentlich vorher schon unhaltbaren Zustand; dazu kamen zahlreiche Vertriebene, die integriert und mit Wohnraum versorgt werden mussten. Frankfurt am Main beispielsweise hatte 1951 mit 563.00o Einwohner_innen schon wieder die Vorkriegsgröße erreicht, bis 1953 waren es bereits 600.00o Einwohner_innen, bis 1963 wuchs die Stadt auf 691.00o (MüllerRaemisch 1998 S 407ff.).

In dieser Situation stellte der moderne Siedlungsbau für viele Deutsche erstmals komfortablen, gesunden und bezahlbaren Wohnraum zur Verfügung 
und vor allem einen, der nicht von investorenüblicher Gewinnmaximierung geprägt war. Altbauten waren im Vergleich dazu nicht nur technisch schlechter ausgestattet, sondern unpraktisch in der Benutzung und oft heruntergekommen. Dazu kommt, dass der moderne Städtebau mit seinen luftigen Gebäudeabständen auf die Erfahrungen in den Feuerstürmen des Bombenkrieges reagierte, in dem die Altstädte für viele Bewohner_innen zur Falle geworden waren. Und auch ideologisch war er denkbar weit vom Heimatschutzstil der Nazis entfernt, was dem nötigen gesellschaftlichen Neubeginn angemessen erschien.

Der mit dem Einsetzen des Wirtschaftswunders wachsende Wohlstand heizte den Wohnraumkonsum quantitativ an, zog aber auch höhere Erwartungen an die Qualität des Wohnraums nach sich. In dieser Situation war die flächenhafte Entwicklung neuer Siedlungen am Stadtrand logisch. Hier war Land relativ problemlos verfügbar und es konnten, anders als in Bestandsgebieten, optimierte Typenhäuser in großer Zahl effizient geplant und gebaut werden, ergänzt um Wohnfolgeeinrichtungen wie Schulen, Kindergärten, Einkaufszentren, Sporteinrichtungen und Kirchen. Die so entstanden Quartiere waren in vieler Hinsicht ein Spiegelbild der bundesrepublikanischen Nachkriegsgesellschaft und ihrer Ideale. Dies zeigt sich beispielsweise in den Illustrationen, die den Bau der Frankfurter Nordweststadt begleiten: Hier wird ein reich belebter öffentlicher Raum dargestellt, in dem eingekauft wird, Kinder spielen und Menschen im Café sitzen. Es werden aber auch gesellschaftliche Stereotype reproduziert: Männer kümmern sich ums Auto, Frauen um die Kinder und das Essen (vgl. hierzu Schwagenscheidt 1964: 24, 78).

\section{Kontext 1968}

Um 1968[1] zeichneten sich verschiedene gesellschaftliche Veränderungen ab, die dazu beitrugen den Siedlungsbau der Nachkriegszeit nachhaltig zu diskreditieren. Dabei konvergieren zwei Linien der Kritik, die im Folgenden kurz analysiert werden sollen.

\section{Generationswechsel und Wertewandel}

Zahlreiche Theoretiker_innen haben sich mit der Verbindung von Konsum und Selbstdarstellung befasst (beispielsweise Veblen 1902, Maslow 1943, Schulze 1992). Ohne deren Theorien und die Kritik daran hier im Detail diskutieren zu können, lässt sich für die Nachkriegszeit in Deutschland doch festhalten, dass die Befriedigung basaler Bedürfnisse weniger selbstverständlich war als heute oder in der Zeit um 1968, als die Kritik am modernen Siedlungsbau laut zu werden begann. Die Generation, die in den 1960er Jahren erwachsen wurde, hatte weder den Krieg noch die Not der unmittelbaren Nachkriegszeit bewusst erlebt. Sie war in einem Umfeld aufgewachsen, in dem kontinuierliche Verbesserungen im Lebensstandard die Norm waren und der Fordismus sich als wenig hinterfragtes Erfolgsmodell etabliert hatte - sei es im Siedlungsbau oder im Einfamilienhaus. Während Konsum für die Welt ihrer Eltern noch durchweg positiv besetzt war und vor allem mit praktischen Erwägungen begründet wurde, entwickelte sich ab dem 
Ende der 1960er Jahre in den (im weitesten Sinne) progressiven Teilen der Gesellschaft eine grundsätzlich konsumkritische Haltung, insbesondere als nach der Ölkrise und dem Bericht des Club of Rome (Meadows et al. 1974) die Kosten des ungebremsten Wachstums unmittelbar spürbar wurden. Andererseits traten beim Konsum selbst der Erlebniswert und das schöne Leben (Schulze 1992: 35ff.) in den Vordergrund.

Für das Wohnen bedeutet dieser Wertewandel, dass von der jüngeren Generation anstatt einer komfortablen, sicheren Wohnumgebung eine interessante, anregende gesucht wurde. Die neu gebauten Siedlungen am Stadtrand konnten das nicht bieten, waren sie doch gebaute Abbilder der gesellschaftlichen Ideale des Fordismus und eines zunehmend als ungerecht empfundenen Rollenmodells - die Frauen waren an ein Leben fernab von Stadt und Arbeitsplatz gebunden. Hieran machte sich die progressive Moderne-Kritik wesentlich fest,[2] denn gerade unter dem Aspekt der Selbstverwirklichung, des schönen Lebens und anregender Erfahrungen jenseits fordistischer Lebensentwürfe wurden die Altbauquartiere der Gründerzeit, dieseit dem zweiten Weltkrieg weiter heruntergekommen waren, für junge Menschen interessant. Sie boten Raum für die kreative Aneignung und Neuinterpretation[3] und waren von einer vergleichsweise gemischten Bevölkerung bewohnt. Die Grundrisse der Altbauwohnungen waren auch geeignet, neue Lebensformen wie beispielsweise Wohngemeinschaften auszuprobieren - in den Familienwohnungen der Nachkriegszeit unvorstellbar.

Aus dem linken Spektrum wurde der Siedlungsbau der Nachkriegszeit daher zunehmend als Fortsetzung des Fordismus im Privatleben kritisiert.[4] Die Siedlungen sind in dieser Lesart einseitig auf die Reproduktion der Arbeitskraft ausgerichtet: Sie schneiden die Bewohner - vor allem die Frauen - intendiert von den politischen und kulturellen städtischen Prozessen ab, die sich in den Altbauquartieren quasi urwüchsig entfalten. Den Siedlungen wird zudem unterstellt, bewusst anregungsarm gestaltet zu sein, um die Profite der Bauwirtschaft zu maximieren; damit zeigen sie die geringe Wertschätzung, die den Bewohner_innen entgegengebracht wird. Dass das Leben in den Siedlungen für viele Bewohner_innen, einen massiven Fortschritt im Leben bedeutete, wird dabei gerne unterschlagen.[5] Im Gegenzug machen die stuckdekorierten Altbauten der Gründerzeit eine erstaunliche Karriere: Sie wurden zum menschenfreundlichen Gegenbild zum modernen Siedlungsbau, trotz der dort noch in den 1960er und 1970er Jahren vorherrschenden schlechten Lebensbedingungen und trotz der Tatsache, dass sie mit dem Ziel der Gewinnmaximierung als Investorenstädtebau errichtet wurden.[6] Bildbände wie Die gemordete Stadt (Siedler/Niggemeyer/Angreß 1964) zeugen von einem romantisch verklärten Bild einer als authentisch angesehenen Urbanität.

Weniger kritisiert oder weniger stark wahrgenommen wird in diesem Zusammenhang, dass die zahlreichen Einfamilienhausgebiete, die in dieser Zeit und mit üppiger staatlicher Förderung entstanden, das überkommene Familienmodell deutlich stärker als die Siedlungen voraussetzten - mit einem, meist männlichen Ernährer und einer Hausfrau, die allenfalls nebenbei arbeiten ging und ansonsten die Erziehung der Kinder organisierte. Dieses Familienmodell wurde und wird steuerlich begünstigt, ebenso wie das Pendeln mit dem Auto, das in dieser Zeit zu einer massentauglichen Option 
wurde und die Voraussetzung für das Leben im suburbanen Einfamilienhaus war und ist. Insofern verwundert es, dass die Siedlungen zum Bezugspunkt der der progressiven Kritik wurden, während es aussieht als seien die Einfamilienhausgebiete weitgehend akzeptiert worden.

\section{Konservative Reaktion}

Die Kritik der eher progressiven, linken Gesellschaftsgruppen und die damit verbundene Umdeutung der Altbauquartiere wäre nicht so wirkmächtig geworden, wenn sie nicht vom anderen Ende des politischen Spektrums unterstützt worden wäre - mit gegensätzlichen Argumenten, aber mit demselben Ergebnis. Mit dem Abwandern der Mittelschichten ins staatlich geförderte Eigenheim und dem Nachzug von in vieler Hinsicht benachteiligten Gruppen in die dadurch freiwerdenden Wohnungen konzentrierten sich ebenfalls ab den späten 1960er Jahren oft mehrfach benachteiligte Menschen in den Siedlungen der Nachkriegszeit.

Dadurch, so die konservative Kritik, würden die Siedlungen zu gesetzlosen Orten, an denen sich Drogensucht, Kriminalität und Gewalt ausbreiten und nicht mehr kontrollieren lassen. Die Hamburger Morgenpost beispielsweise schrieb am 3. April 1978: „Wohnsilos mit über 1000 Familien sind Brutstätten von Verbrechen, Krankheiten, Alkohol- und Drogensucht! Die Kriminalität in solchen Betonburgen ist sieben- bis zehnmal höher als in Gegenden mit normaler Bebauung." Der Beststeller Wir Kinder vom Bahnhof Zoo (Christiane F. et al. 1978) und der anschließende Film verorteten all dies medienwirksam in der Berliner Gropiusstadt. Ohne die Ursachen für die statistischen Auffälligkeiten näher zu beleuchten, verfestigt sich so das Bild von Siedlungen der Nachkriegsmoderne als Orte der Devianz und als Gefahr für die öffentliche Ordnung.

Selbstverständlich verschont auch die konservative Kritik die Einfamilienhausgebiete, festigen sie doch traditionelle Rollenbilder und Lebensstile. Trotz aller praktischen Schwierigkeiten in der Organisation des Alltags eine bis heute gesellschaftlich akzeptierte Wohnform.

\section{Neuer Konsens}

So unterschiedlich die Linien der Kritik sind, im Ergebnis führten Sie zu einer bis heute fortwirkenden negativen Sicht auf den Siedlungsbau der Nachkriegsmoderne und einer bisweilen klischeehaften, positiven Sicht auf den Altbaubestand der Gründerzeit. Darüber hinaus folgten ihnen auch konkrete, politische Veränderungen - vor allem das Ende des großmaßstäblichen Siedlungsbaus und die Hinwendung zur Europäischen Stadt, kleinteilig parzelliert und nutzungsgemischt. Zu den konkreten Veränderungen gehören aber auch Förderprogramme, die den Erhalt, die Ertüchtigung und die Aufwertung des Altbaubestandes zum Ziel hatten und haben. Sanierung heißt heute fast immer, dass mit dem Bestand gearbeitet wird, nicht dass er großflächig abgebrochen und ersetzt wird. In vielkritisierten modernen Siedlungen wurden bestandsorientierte Erneuerungsprogramme zunächst nicht vorgesehen, vielmehr wurde der Bau in manchen Fällen sogar gestoppt, was zu einer weiteren Verschärfung bestehender Probleme führte: entweder 
weil Bewohner_innen fehlten, die die bereits gebaute Infrastruktur hätten auslasten können (beispielsweise in der neuen Stadt Wulfen), oder weil die geplante Infrastruktur nicht mehr realisiert wurde (etwa der S-BahnAnschluss der Hamburger Siedlung Steilshoop). Erst seitdem 1999 das Förderprogramm „Soziale Stadt“ aufgelegt wurde, werden die strukturellen Defizite der modernen Siedlungen in den Blick genommen und teilweise sehr erfolgreich behoben.

Projekte wie die „IBA Berlin 1977-1984“ haben dazu beigetragen, dass die bestandsorientierte Weiterentwicklung von Gründerzeitquartieren zu einem allgemein anerkannten Modus der Stadtentwicklung geworden ist und dass erhebliche Mengen öffentlicher Gelder in Projekte dieser Art geflossen sind. Erst mithilfe umfangreicher staatlicher Förderung wurden heruntergekommene Altbauwohnungen in entkernten und begrünten Blockstrukturen zu komfortablen Behausungen, die mit dem modernen Siedlungsbau nicht nur mithalten konnten, sondern ihm bestenfalls sogar überlegen waren. Erst im Zuge dieser Entwicklung wurde die sanierte, gründerzeitliche Altbauwohnung, möglichst mit erhaltener repräsentativer Original-Ausstattung, eine allgemein akzeptierte Alternative zum Eigenheim im Grünen.

Die konkrete Ausgestaltung von Stadterneuerungsprojekten zeigt auch, dass jenseits des Erhalts von historischer Bausubstanz immer auch wirkmächtige Bilder erzeugt wurden. Diese spiegeln weniger eine historische Wahrheit wider als die zum jeweiligen Zeitpunkt vorherrschenden Idealvorstellungen von Stadt, Urbanität und dem schönen Leben. Vorher-nachher-Bilder zeigen, dass erheblicher Aufwand getrieben wurde, um vermeintlich unhistorische Elemente und nachträgliche Veränderungen zu eliminieren (Vinken 2010). Im Rahmen dieser Debatte unterstelle ich, dass es dabei immer auch darum ging, eine attraktive Kulisse für das Leben urbaner Mittelschichten zu schaffen.

Beides, das Eigenheim und die gründerzeitliche Altbauwohnung, erlauben ein relativ hohes Maß an Unabhängigkeit in der Lebensgestaltung, wenn auch in unterschiedlicher Art und mit unterschiedlichen Mitteln. Der Siedlungsbau hingegen verkörpert nach wie vor ein fordistisches Gesellschaftsmodell, das als überholt angesehen wurde und für die Eliten seit 1968 nicht mehr relevant war, weil es sich nicht mit einem hohen Erlebniswert verbinden ließ. Damit ist im Diskurs eine nachhaltige und in weiten Teile der Gesellschaft verankerte Verknüpfung von Siedlungsbau der Nachkriegsmoderne und sozialen Problemlagen entstanden. Die Kausalitäten werden dabei manchmal vertauscht und oft entsteht der Eindruck, dass die Siedlungen massive soziale Probleme verursachen. Dass Probleme sich dort gerade wegen der vorherrschenden Entwertung dieser Siedlungsform und der damit verbundenen relativen Zugänglichkeit für benachteiligte Gruppen konzentrieren, wird hingegen gerne übersehen.

Die Tatsache, dass Stadtforscher_innen sich heute wissenschaftlich mit der Zeit um 1968 befassen kann man auch als einen Hinweis darauf lesen, dass die zwingende Identifikation des Siedlungsbaus der Nachkriegszeit mit fordistischen Lebensmodellen historisch zu werden beginnt. Dies eröffnet die Möglichkeit, diese Art von Stadt abermals neu zu interpretieren und neu zu besetzen, wie Reckwitz es beschrieben hat (2012), unabhängig von den ursprünglichen Intentionen und Rahmenbedingungen. Damit können auch neue Konkurrenzen und Konflikte entstehen, die denen, die 
in Gründerzeitquartieren zu beobachten sind, ähneln - und die, wie schon die Umwertung ab 1968, vorwiegend auf der Ebene kultureller Differenzen ausgetragen werden könnten. Die Verdrängung aus den Wohnungen durch kapitalstärkere Gruppen ist dabei nur ein mögliches Konfliktfeld, die Deutungshoheit über den Raum, unterschiedliche Aneignungsprozesse und Nutzungsmuster ein weiteres. Es gibt jedenfalls keinen Grund anzunehmen, dass die Siedlungen der Nachkriegszeit von der Wiederentdeckung und den damit verbundenen Umbrüchen verschont bleiben, die die Stadt der Gründerzeit bereits erfahren hat.

\section{Endnoten}

[1] Die Kritik am modernen Siedlungsbau setzt schon vor 1968 ein und dauert nach 1968 weiter an. Dennoch ist das Jahr 1968 so eng mit längerfristigen politischen und gesellschaftlichen Umbrüchen verbunden, dass es hier als Referenz genutzt wird.

[2] Zahlreiche deutsche und internationale Publikationen beschäftigen sich mit dem Versagen von Städtebau und Architektur der Moderne. Dabei ist der Duktus bisweilen polemisch und die Argumentation anekdotisch. Beispielhaft genannt seien hier nur einige: Die gemordete Stadt (Siedler/Niggemeyer/Angreß 1964), Die Unwirtlichkeit unserer Städte (Mitscherlich 1965), Profitopolis (Lembrock 1971), Form Follows Fiasko (Blake 1977)), Umwelt Stadt (Conrads 1974) oder From Bauhaus to Our House (Wolfe 1981).

[3] Andreas Reckwitz hat in seinem Buch Die Erfindung der Kreativität (2012) ausführlich dargestellt, wie kreative Prozesse heute mit Auswahl, Neuinterpretation und kultureller Aneignung korrespondieren und wie sich dies auf die Entwicklung von Quartieren und städtischen Räumen auswirkt (Reckwitz 2012: 287ff).

[4] Noch 2009 kritisiert beispielsweise Dieter Hoffmann-Axthelm in einem Interview, es habe „damals in Deutschland ein neues Gesellschaftsbild geherrscht: das einer staatlich organisierten Massengesellschaft. Man glaubte, der Staat sei verpflichtet, die Vergesellschaftung aller Lebensbezüge vorzunehmen, die vorher in der Verantwortung der Individuen lag“ (Braum/Welzbacher 2009: 42). Für einen Zeitzeugen, der eine relativ behütete Jugend im Berliner Hansaviertel verbracht hatte, ist das nachvollziehbar, aber Hoffmann-Axthelm unterschlägt dabei, dass die Siedlungen für viele der Bewohner auch heute noch Heimat ist, zu der sie ein durchaus emotionales Verhältnis pflegen.

[5] Die dokumentarischen Fotografien, die Heinrich Kuhn in den 1960er Jahren von Wohnungen in Häusern machte, die im Rahmen von Sanierungsvorhaben abgebrochen werden sollten, zeigen, wie unerfreulich die Realität in den Altbaubeständen noch lange nach dem Kriegsende war (Krüger 2014).

[6] Werner Hegemann hat in seiner Polemik Das steinerne Berlin (1930) alle Probleme der investorengesteuerten Berliner Stadtentwicklung der Gründerzeit ausführlich dargestellt.

\section{Autor_innen}

Maren Harnack hat Architektur, Stadtplanung und Sozialwissenschaften in Stuttgart, Delft und London studiert. Ihre Arbeitsschwerpunkte sind Siedlungs- und Städtebau der Nachkriegsmoderne.

maren.harnack@fb1.fra-uas.de 


\section{Literatur}

Blake, Peter (1977): Form Follows Fiasko. Why Modern Architecture Hasn't Worked. Boston/Toronto: Little, Brown and Company.

Conrads, Ulrich (1974): Umwelt Stadt. Argumente und Lehrbeispiele für eine humane Architektur. Reinbek bei Hamburg: Rohwolt.

Christiane F. et al. (2009 [1978]): Wir Kinder vom Bahnhof Zoo. Hamburg: Gruner \& Jahr.

Hegemann, Werner (1963 [1930]): Das steinerne Berlin. Basel: Birkhäuser.

Krüger, Sabine (Hg.) (2014): Armutszeugnisse. Westberlin vor der Stadtsanierung in den sechziger Jahren. Fotografien von Heinrich Kuhn. Berlin: Edition Braus.

Lehmbrock, Josef (Hg.) (1971): Profitopolis oder: Der Mensch braucht eine andere Stadt. München (Ausstellungskatalog).

Maslow, Abraham (1943): A theory of human motivation. In: Psychological Review 50, 370-396. http://psychclassics.yorku.ca/Maslow/motivation.htm (letzter Zugriff am 16.8.2018).

Meadows, Donella et al. (1974): The Limits to Growth. A Report for the Club of Romes Project on the Predicament of Mankind. New York: Universe Books.

Mitscherlich, Alexander (1965): Die Unwirtlichkeit unserer Städte. Eine Anstiftung zum Unfrieden. Frankfurt am Main: Suhrkamp.

Müller-Raemisch, Hans-Reiner (1998): Frankfurt am Main. Stadtentwicklung und Planungsgeschichte seit 1958. Frankfurt am Main / New York: Campus

Reckwitz, Andreas (2012): Die Erfindung der Kreativität. Zum Prozess gesellschaftlicher Ästhetisierung. Berlin: Suhrkamp.

Schwagenscheidt, Walter (1964): Die Nordweststadt. Idee und Gestaltung. Stuttgart: Karl Krämer Verlag.

Schulze, Gerhard (1992): Die Erlebnisgesellschaft. Kultursoziologie der Gegenwart. Frankfurt am Main/New York: Campus.

Siedler, Wolf Jobst / Niggemeyer, Elisabeth / Angreß, Gina (1967 [1963]): Die gemordete Stadt. Abgesang auf Putte und Straße, Platz und Baum. Berlin u. a.: Herbig.

Veblen, Thorstein (2013 [1902]): The Theory of the Leisure Class. www.gutenberg.org/ ebooks/833, letzter Zugriff am 16.8.2018.

Vinken, Gerhard (2010): Zone Heimat. Altstadt im modernen Städtebau. Berlin: Deutscher Kunstverlag.

Welzbacher, Christian (im Gespräch mit Dieter Hoffmann-Axthelm, 2009): Die katastrophale Utopie. Planungswirtschaft und Sozialdogmatismus. In: Michael Braum / Christian Welzbacher (Hg.), Nachkriegsmoderne in Deutschland. Eine Epoche weiterdenken. Berlin: Jovis, 36-51.

Wolfe, Tom (2009 [1981]): From Bauhaus to Our House. New York: Picador. 
